\title{
Validity of maternal reporting of breast feeding history and the association with blood lipids in 17 year olds in Jerusalem
}

\begin{abstract}
J D KARK, ${ }^{1}$ G TROYA, ${ }^{2}$ Y FRIEDLANDER, ${ }^{3}$ P E SLATER, ${ }^{4}$ AND Y STEIN ${ }^{3}$
From the Departments of Social Medicine ${ }^{1}$ and Medical Ecology, ${ }^{4}$ Hebrew University-Hadassah School of Public Health and Community Medicine and Hadassah Medical Organization, Ein Karem, Jerusalem, Israel, the Division of Sociomedical Research, ${ }^{2}$ Ministry of Health, Quito, Ecuador, and the Lipid Research Clinic, ${ }^{3}$ Department of Internal Medicine B, Hadassah University Hospital, Ein Karem, Jerusalem, Israel
\end{abstract}

SUMMARY The validity of mothers' reporting of the breast feeding history of their children more than 20 years after their birth was studied in a Jerusalem population. Among 74 study subjects, duration of breast feeding as ascertained from an interview was well correlated with that recorded in mother and child health clinic charts. Concordance was of similar magnitude in subgroups of ethnicity, mother's education, family size, and sex of child. Duration of breast feeding in 101. youngsters was inversely associated with plasma cholesterol in 17 year old girls, though not in boys, which was statistically significant on univariate analysis and of borderline significance on multivariable analysis. Among 17 year old boys, though not in girls, a statistically significant inverse association for plasma triglyceride was apparent on multivariable analysis. Reported breast feeding history derived from interview of mothers may be a useful instrument for study of possible long term effects of breast feeding in their adolescent or adult progeny.

This investigation was prompted by the report of Marmot $e t$ al who showed, in the framework of a historical prospective study, that adults in their 30s who had been exclusively breast fed during infancy for five months or longer had lower plasma cholesterol concentrations than those who had been bottle fed. ${ }^{1}$ This was more evident for girls than boys. A study of this association necessitates either the availability of an adult cohort with well documented breast feeding data during infancy (an unusual situation), or the patience (and longevity) to undertake a 20-30 year prospective study beginning with a cohort of newborns in the present, or the use of a retrospective breast feeding questionnaire and current cholesterol estimation in a study of adults. The last approach requires validation of the breast feeding history.

We selected the third option. Plasma lipid and lipoprotein data on a large population of 17 year olds were available from the Jerusalem Lipid Research Clinic Study. We decided to interview the mothers of the 17 year olds (then aged 20 to 22) to derive breast feeding histories for those subjects whose mother and child clinic records had remained intact over the ensuing 20 to 22 years. Self reporting of breast feeding by adults was found to be of low validity ${ }^{1}$ and was thus not attempted.

The objectives of this study were twofold:

(1) To validate a breast feeding history questionnaire applied to mothers about 20 years after the birth of their children against available routine records from mother and child clinics.

(2) To conduct a preliminary study of the relation of breast feeding with plasma lipid concentrations in a sample of Jerusalem residents aged 17. The lipids measured were plasma cholesterol, high density lipoprotein cholesterol, and triglycerides.

Should the retrospective breast feeding information prove to be sufficiently valid, we would be able in a subsequent study to pursue the question of a breast feeding blood lipid association in a large sample of subjects examined by the Jerusalem Lipid Research Clinic.

\section{Methods}

The Jerusalem Lipid Research Clinic Study and initial prevalence findings have been described in 218 
detail. ${ }^{2}$ All 17 year old Jerusalem residents attending their premilitary service health examination from 1976 to 1978 were invited to participate. The method of sampling, response characteristics, and the distribution of plasma lipids and lipoproteins among the 864617 year old participants (4960 boys and 3686 girls) have been published..$^{3}$

Unfortunately, mother and child clinic records for the birth years of our cohort of Jerusalem residents (1959-61) had been destroyed in all but two of the numerous clinics in the city. In one of these clinics a $5 \%$ systematic sample of records had been saved whereas in the other most records had been maintained. Attendance at mother and child clinics is universal in Israel. The search was done by matching the names and dates of birth of subjects born between 1959 and 1961 who attended the two clinics with those examined by the Jerusalem Lipid Research Clinic at age 17 . Records were found for only 125 subjects.

Of the 125 mothers, 104 (83\%) were interviewed, 94 by home visit and 10 by telephone. Five mothers refused, seven were living abroad, and nine could not be located. The two central questions of interest in the interview were "Did you breast feed your son/daughter when he/she was a baby?" and "For how long did you breast feed him/her?" Interviewers were unaware of the mother and child clinic record data on breast feeding. Information on family size, birth order, mother and father's education, mother and father's occupation, mother and father's and grandfather's country of birth, breast feeding of other children in the family, and age at which liquid cows' milk or powdered milk was introduced was also gathered. One of the 104 mothers interviewed could not recall the breast feeding history and in one case the interview form was unclear. Complete breast feeding data were found in the clinic records for only 84 of the 125 babies (67\%); data were incomplete in 23 and missing in 18. A breast feeding record was deemed complete if there was a recorded entry for non-breast feeding or for cessation of breast feeding, or if the baby had been breast fed for more than six months in the absence of definitive data on weaning.

The validation study was based on 74 subjects with complete data from both sources. The association of plasma lipids in 17 year olds with breast feeding during infancy was analysed using both record data (84 subjects) and interview data (101 subjects after exclusion of one boy with no lipid data).

Plasma lipids (plasma cholesterol, high density lipoprotein cholesterol, and triglycerides) were measured according to the Lipid Research Clinic Program protocol ${ }^{5}$ after a 12 hour fast. Seventy four per cent of the boys and $82 \%$ of the girls reported fasting for at least 12 hours before examination. ${ }^{3}$
Weight and height were measured with subjects wearing underclothes. Quetelet's index $\left(\mathrm{kg} / \mathrm{m}^{2}\right)$ was used as a measure of body mass. A six point social class scale based on the occupation of the head of the household was used. ${ }^{3}$ Country of origin was classified into two groups based on country of birth of the father (Israel/Europe and North Africa/Asia).

Spearman rank order correlations and linear regression were used to evaluate the association between reported and recorded duration of breast feeding. The Kappa statistic ${ }^{6}$ and measures of sensitivity and specificity were used to describe the degree of concordance between dichotomised data from the two sources.

Association of plasma lipids with duration of breast feeding was examined by inspecting scattergrams, by Spearman rank order correlation, and by multiple linear regression and analysis of covariance to control for possible confounding factors. Because of apparent unequal variance (heteroscedasticity), a square root transformation of cholesterol and triglyceride values was also used in the regression analyses.

\section{Results}

\section{PATTERNS OF BREAST FEEDING}

Table 1 gives the mean, median, and standard deviations of both recorded and reported duration of breast feeding. About $10 \%$ of the mothers did not breast feed their babies at all, while the median duration of breast feeding was about 13 weeks. Children of less educated mothers, of mothers of North African or Asian origin and coming from larger families were breast fed for longer periods (table 1). Other than breast milk the major milk source for babies was cows' milk. The median age at which complementary milk (mainly liquid cows' milk) was introduced was about 5 weeks according to the records and 7 to 8 weeks according to the interview. Records showed that by 12 weeks over $80 \%$ of babies received milk other than breast milk as the sole or complementary milk source. Use of powdered milk was apparently uncommon. Any feeding with powdered milk was recorded in $14 \%$ of all charts found and was reported by $20 \%$ of the mothers interviewed.

\section{VALIDITY OF BREAST FEEDING HISTORY}

Patterns of breast feeding were similar when both data sources were compared. The means and standard deviations of the interview data tended to be higher than those of the records whereas the median values were similar. The small difference in means may reflect possible truncation of breast feeding duration in seven records defined as 
complete (see methods) or possible overreporting of duration in the interview for the long term breast feeders. Overall agreement between the two sources of information was generally high when evaluated by correlation analysis (table 1). The Spearman correlation was 0.86 (and the Pearson correlation $0 \cdot 82$ ). The intercept ( $\pm S E$ ) for the regression of the interview data on recorded data was near to the origin $(0 \cdot 7 \pm 2 \cdot 1$ weeks) and the slope $( \pm S E)$ close to unity $(1.078 \pm 0.088)$. Omission of the seven possibly truncated cases did not alter the correlations whereas the change in regression coefficients was trivial. (Use of all mother and child clinic records that had any breast feeding data-that is, inclusion of 12 additional records defined as incomplete-reduced the correlation with the interview data to 0.76 . Regression coefficients were unchanged.)

The concordance was examined within the sex, ethnic group, mother's education, and family size strata. In all categories, notwithstanding the rather small numbers of subjects in the subgroups, agreement was consistently high (table 1 ). When the data were stratified by pairs of variables (sex and mother's education; sex and number of children in family (classified into two groups of one to three children and four or more); ethnic origin and number of children; and mother's education and number of children) the Spearman correlation coefficients ranged from 0.72 to 0.96 . Pearson correlations were similar.

In 36 of the 74 subjects the recorded breast feeding duration was shorter than that reported, in 10 cases identical, and in 28 longer. After exclusion of the seven possibly truncated records, 30, 10, and 27 subjects remained in the three respective categories.
On arbitrary dichotomisation of duration of breast feeding into long ( $>5$ months) and short ( $\leq 5$ months), the percentage agreement between reported and recorded data was $91 \%$ and the Kappa statistic was 0.79 . The sensitivity of the reported data for correctly identifying long term breast feeders was $91 \%$ and the specificity $90 \%$. When breast feeding duration was divided into short term ( $<2$ months) and long ( $\geq 2$ months), the percentage agreement was $85 \%$, Kappa was $80 \%$, the sensitivity of correctly classifying short term breast feeders was $86 \%$, and the specificity $84 \%$.

Thus whether breast feeding was treated as a continuous or a categorical variable, the reported history 20 years later appears to be a fairly valid estimate of the recorded data.

The correlation between reported and recorded first use of complementary milk (liquid cows' milk or powdered milk) was low $(r=0.16$ and after excluding two obvious outliers, $\mathbf{0 \cdot 4 6}$ ). Concordance on use of powdered milk was extremely low. Of 11 records that indicated use of powdered milk, only two were identified in the interview.

REPRESENTATIVENESS OF THE STUDY POPULATION

Characteristics of the breast feeding study population were compared with those of the total Jerusalem Lipid Research Clinic sample (table 2). Overall, there was little difference in weight, height, body mass, and lipid values between the ascertained을 sample for the present study and the total sample.? The ethnic composition was nearly identical for the broad groupings used. No differences were

Table 1 Spearman rank order correlation of duration of breast feeding derived from mother and child health clinic records with reported breast feeding history obtained by interview 20 to 22 years later according to sex, education of mother, country of origin, and family size

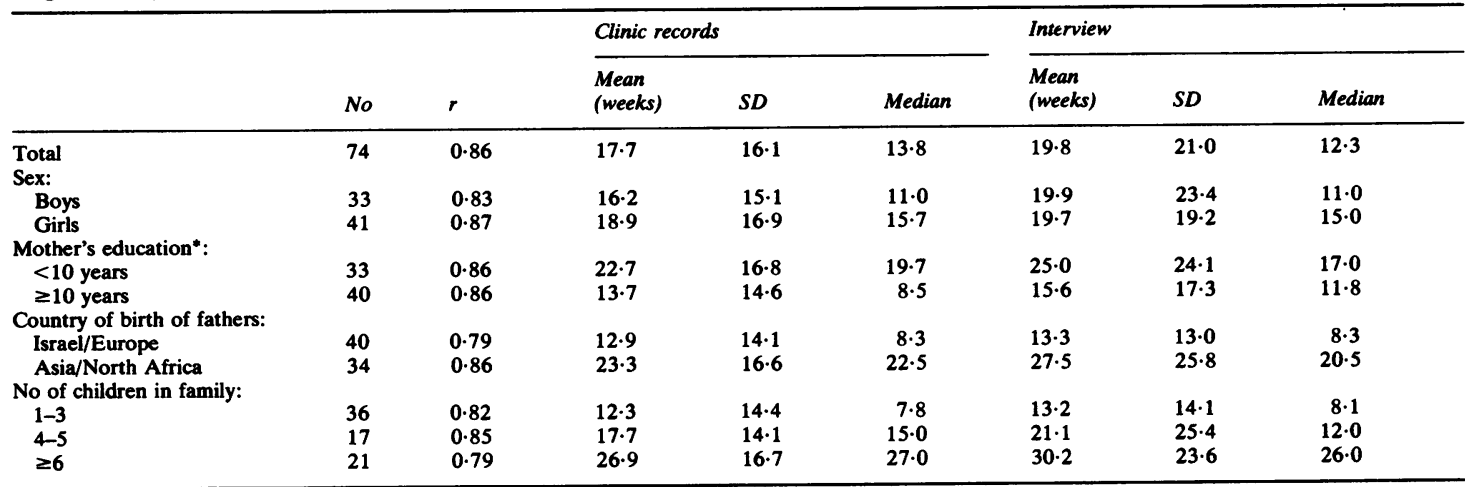

*One person missing. 
statistically significant at the $\alpha=0.05$ level.

Thus although the study sample was small and was not randomly ascertained but was drawn from the two neighbourhood clinics in which records were extant, it seems to be reasonably representative of the diverse source population in terms of the characteristics that were compared.

Table 2 Ethnic, anthropometric, and plasma lipid characteristics of the study sample in comparison with the Jerusalem Lipid Research Clinic study population, according to the source of the breast feeding data: mother and child clinic records and interview (mean $\pm S D$ )*

\begin{tabular}{lccc}
\hline & MCH records & Interview & LRCVisit I \\
\hline & Boys & & \\
No & 38 & 50 & 4960 \\
Israel/Europe (\%) & 50 & 50 & $50 \cdot 4$ \\
Asia/North Africa (\%) & 50 & 50 & $49 \cdot 6$ \\
Height (cm) & $173 \cdot 0 \pm 6 \cdot 1$ & $171 \cdot 7 \pm 7 \cdot 4$ & $173 \cdot 1 \pm 6 \cdot 7$ \\
Weight (kg) & $62 \cdot 9 \pm 8 \cdot 9$ & $61 \cdot 5 \pm 7 \cdot 7$ & $63 \cdot 4 \pm 9 \cdot 8$ \\
Quetelet index (kg/m) & $21 \cdot 0 \pm 2 \cdot 5$ & $20 \cdot 9 \pm 2 \cdot 4$ & $21 \cdot 1 \pm 2 \cdot 8$ \\
Plasma cholesterol (mg/dl) & $133 \pm 25$ & $133 \pm 22$ & $133 \pm 24$ \\
Plasma HDL-cholesterol (mg/dl) & $40 \pm 8$ & $41 \pm 9$ & $42 \pm 10$ \\
Plasma triglyceride (mg/dl) & $83 \pm 39$ & $80 \pm 43$ & $77 \pm 35$ \\
& & & \\
No & Girls & & \\
Israel/Europe (\%) & 46 & 52 & 3686 \\
Asia/North Africa (\%) & 52 & 50 & $52 \cdot 7$ \\
Height (cm) & 48 & 50 & $47 \cdot 3$ \\
Weight (kg) & $161 \cdot 2 \pm 6 \cdot 1$ & $161 \cdot 2 \pm 5 \cdot 7$ & $161 \cdot 3 \pm 6 \cdot 1$ \\
Quetelet index (kg/m) & $55 \cdot 2 \pm 6 \cdot 5$ & $55 \cdot 0 \pm 5 \cdot 8$ & $56 \cdot 4 \pm 8 \cdot 2$ \\
Plasma cholesterol (mg/dl) & $21 \cdot 2 \pm 2 \cdot 3$ & $21 \cdot 2 \pm 2 \cdot 1$ & $21 \cdot 7 \pm 2 \cdot 8$ \\
Plasma HDL-cholesterol (mg/dl) & $158 \pm 28$ & $155 \pm 29$ & $151 \pm 28$ \\
Plasma triglyceride (mg/dl) & $50 \pm 10$ & $51 \pm 10$ & $49 \pm 11$ \\
& $84 \pm 43$ & $79 \pm 43$ & $76 \pm 36$ \\
\hline
\end{tabular}

*Weight and height missing in six girls with records and with interview data, and in 228 boys and 406 girls in the Jerusalem Lipid Research Clinic visit 1 sample; lipid values missing for one boy with interview data.

BREAST FEEDING/LIPID ASSOCIATIONS

Correlation analysis (table 3)

Sex specific Spearman rank correlations of breast feeding duration (in weeks) with the various lipids were computed. (Pearson product moment coefficients tended to be slightly higher for most comparisons.) We were hesitant to pool the sexes, particularly since a previous study had suggested a sex-breast feeding interaction on cholesterol values. ${ }^{1}$

A statistically significant inverse association of duration of breast feeding with plasma cholesterol was evident in girls, both for recorded and reported data $(r=-0.28, p=0.030 ; r=-0.26, p=0.031$ respectively). In boys the associations were inconsistent and did not differ significantly from zero.

Associations with high density lipoprotein cholesterol (HDL-C) were weak and inconsistent. The proportion of HDL-cholesterol in total cholesterol was positively correlated with duration of breast feeding in girls for reported data only $(r=0 \cdot 20, p>0.05)$.
Table 3 Spearman correlations of duration of breast feeding with plasma cholesterol (CHOL), plasma triglyceride (TG), plasma high density lipoprotein cholesterol $(H D L-C)$, and the proportion of $H D L-C$ in CHOL according to sex and source of breast feeding data (records or interview)

\begin{tabular}{llllll}
\hline & \multicolumn{2}{l}{ Records } & & \multicolumn{2}{l}{ Interview } \\
\cline { 2 - 3 } \cline { 5 - 6 } & Girls & Boys & & Girls & Boys \\
\hline No & 46 & 38 & & 52 & 49 \\
CHOL & $-0.28^{*}$ & 0.10 & & $-0.26^{*}$ & -0.07 \\
TG & -0.003 & -0.18 & & -0.18 & -0.23 \\
HDL_C & -0.17 & -0.02 & & -0.02 & -0.01 \\
HDLC/CHOL & 0.07 & -0.08 & & 0.20 & 0.04 \\
\hline
\end{tabular}

"p $<0.05$

For triglyceride, stronger inverse associations were noted in boys than in girls. Correlations of a similar magnitude were seen for both recorded and reported data in boys $(r=-0.18$ and $-0 \cdot 23$, respectively, $p>0.05)$. Associations were less consistent in girls. Exclusion of boys who had fasted for less than 12 hours before giving blood reduced the correlations slightly. The inverse correlation was strong in non-fasters of both sexes, exceeding -0.5 for both parametric and non-parametric measures.

Weak positive correlations (from 0.05 to 0.15 ) were seen for the duration of breast feeding and Quetelet's index of body mass in both sexes (data not shown).

\section{Multivariate analysis (table 4)}

The association of body mass index, ethnicity, and social class with lipid concentrations in 17 year olds in the Jerusalem Lipid Research Clinic Study has been shown. ${ }^{7}$ Within our data set body mass index was associated with plasma cholesterol in girls (Pearson $\mathbf{r}=\mathbf{0} \cdot 30$ ) and with plasma triglyceride in boys (Pearson $r=0.23$ ), and showed a weak positive association with breast feeding. Ethnicity, mother's education, and social class were strongly associated with breast feeding. These variables were considered as potential confounders. Mother's education was included only in the analyses based on interview data. interview data.

Univariate and multivariate regression coefficients for the regression of plasma cholesterol and of triglyceride ( $\log _{e}$ transformation) on duration of breast feeding are seen in table 4. Regressions were run in two ways: (a) in forward stepwise mode with the programme selecting variables for entry according to the highest partial correlation coefficient until the peak adjusted "explained" variance $\left(R^{2}\right)$ was reached or (b) until all variables entered the equation. Inspection of the bivariate scattergrams suggested an inverse curvilinear relation of plasma cholesterol with duration of breast 
Table 4 Univariate and multivariate coefficients (b) for the regression of plasma cholesterol and plasma triglyceride $\left(\log _{e}\right.$ transformation) on duration of breast feeding, separately for record and interview data and for sex

\begin{tabular}{|c|c|c|c|c|c|c|c|c|c|c|}
\hline \multirow{3}{*}{$\begin{array}{l}\text { Dependent variable } \\
\text { Study group }\end{array}$} & \multirow[b]{3}{*}{ No } & \multicolumn{3}{|l|}{ Univariate* } & \multicolumn{6}{|c|}{ Multivariate ${ }^{\dagger}$} \\
\hline & & \multirow[b]{2}{*}{$b$} & \multirow[b]{2}{*}{$S E$} & \multirow[b]{2}{*}{$R^{2}$} & \multicolumn{3}{|c|}{ Regression No $1 \ddagger$} & \multicolumn{3}{|c|}{ Regression No 28} \\
\hline & & & & & $b$ & $S E$ & $\begin{array}{l}\text { Adjusted } \\
R^{2}\end{array}$ & $b$ & $S E$ & $R^{2}$ \\
\hline \multicolumn{11}{|l|}{$\begin{array}{l}\text { Cholesterol } \\
\text { Boys }\end{array}$} \\
\hline Record data & 38 & 0.012 & 0.287 & 0.00 & - & - & - & 0.033 & 1.66 & 0.015 \\
\hline Interview data & 49 & -0.196 & 0.152 & 0.034 & - & - & - & -0.196 & 0.162 & 0.120 \\
\hline \multicolumn{11}{|l|}{ Girls } \\
\hline Record data & 46 & $-0.497^{*}$ & 0.244 & 0.087 & $-0.573^{*}$ & 0.234 & $0 \cdot 151$ & $-0.539^{*}$ & 0.267 & 0.222 \\
\hline Interview data & 52 & $-0.430^{*}$ & $0 \cdot 195$ & 0.088 & -0.351 & $0 \cdot 203$ & $0 \cdot 161$ & -0.366 & 0.205 & 0.222 \\
\hline \multicolumn{11}{|l|}{$\begin{array}{l}\text { Triglyceride } \\
\text { Boys }\end{array}$} \\
\hline Record data & 38 & -0.00545 & 0.00466 & 0.037 & -0.00527 & 0.00461 & 0.049 & -0.00517 & 0.00470 & 0.128 \\
\hline Interview data & 49 & -0.00471 & 0.00245 & 0.073 & $-0.00564^{*}$ & 0.00242 & $0 \cdot 108$ & $-0.00579^{*}$ & 0.00263 & 0.174 \\
\hline \multicolumn{11}{|l|}{ Girls } \\
\hline Record data & 46 & -0.00050 & 0.00384 & 0.00 & - & - & - & -0.00049 & 0.00398 & 0.055 \\
\hline Interview data & 52 & -0.00249 & 0.00333 & 0.011 & - & - & - & -0.00211 & 0.00371 & 0.076 \\
\hline
\end{tabular}

* $p \leqslant 0.5$.

†In the univariate analysis a linear term for the duration of breast feeding was the only independent variable whereas in the multivariate mode Quetelet index, ethnic origin, mother's education, and father's social class were added. $b$ is the regression slope coefficient, SE the standard error of $b$, and $\mathbf{R}^{2}$ the proportion of total variance "explained" by variables in the regression equation. For six girls with missing data for weight and height the mean Quetelet values of their respective country of origin group were substituted.

¥Stepwise regression allowed to proceed to maximum adjusted $\mathbf{R}^{\mathbf{2}}$; in four instances duration of breast feeding did not enter.

8 All variables introduced into the regression.

feeding in girls and of plasma triglyceride with breast feeding in boys. Introduction into the regression of a quadratic term for duration of breast feeding, or alternatively of a threshold term, did not significantly improve the fit. In addition there appeared to be heteroscedasticity with evidently greater variance of cholesterol (in girls) and triglyceride (in boys) at the shorter durations of breast feeding. Use of a square root transformation of the lipid variables made no added contribution. Thus these terms were not used.

Univariate slope coefficients for the regression of cholesterol on breast feeding in girls were negative in sign, statistically significant, and similar in magnitude for recorded and reported breast feeding data. In boys the relation was weaker and less consistent. Multivariate adjustment for covariates resulted in a small increase in the slope coefficient for recorded data in girls and a moderate reduction in the coefficient for reported data. Both coefficients were of borderline statistical significance. For triglyceride in boys the coefficients for both data sources were of similar magnitude for both the univariate and multivariate analyses, although statistically significant only for the reported breast feeding data. In girls there was no evident association.

Duration of breast feeding was dichotomised at the median of boys ( $\leq 12$ and $\geq 13$ weeks) and also at the median of girls ( $\leq 16$ and $\geq 17$ weeks). Lipid associations were analysed by analysis of covariance controlling for ethnic group and for body mass index. Using both cutpoints, univariate and multivariate differences in cholesterol means and of $\log$ 象 transformed triglyceride means were consistent with the trends seen in the regression analyses but were not statistically significant at the $\alpha=0.05$ level There was a suggestion of an ethnic group/breas feeding interaction on cholesterol in both sexes.

\section{Discussion}

REPRESENTATIVENESS OF THE STUDY SAMPLE Our study sample included the diverse ethnic and social class strata that characterise the Jewish population of Jerusalem and of Israel. Anthropometric and plasma lipid characteristics were quite similar to those of the source population. Thus although this was not a randomly selected sample, it is unlikely that it differs substantially from the Lipid Research Clinic study population from which it was drawn.

In terms of the small percentage of babies never breast fed the median age at weaning, ethnic differences in breast feeding practices, and age at introduction of supplementary food the patterns of the study population closely reflected survey findings of $1956^{8}$ and $1958 .^{\circ}$

\section{VALIDITY OF REPORTED BREAST FEEDING} HISTORY

The overall high validity of breast feeding history as reported by the mother in a face to face or telephone interview more than 20 years after the birth of her child is impressive. Within the limitations of a small 
sample, validity appeared to be generally non-differential in that the various groupings categorised according to sex of the child, number of children in the family, ethnic origin, and mother's education showed similarly high concordance. Eighty per cent of interviewed mothers reported breast feeding all their children. If mothers maintained similar patterns for all their children this could have enhanced recall and may partly explain the high validity. Interview of mothers about their breast feeding practices appears to be a useful instrument that is applicable to widely differing educational and cultural groupings in the Jewish population in Israel.

We are aware of only limited data in published reports relating to the validity of breast feeding recall. In a well-baby clinic poptation in Oslo ${ }^{10}$ the correlations of duration of breast feeding ascertained from records, and from interview of mothers six months and eight years after their babies' birth, were 0.96 and 0.77 respectively. A study of parents of 3 year olds in New York (44 mothers and 39 fathers, mostly college graduates) ${ }^{11}$ showed that whether the baby was initially breast or bottle fed was accurately recalled by both parents whereas duration of breast feeding was better remembered by mothers. Of the various child rearing practices analysed in both studies, breast feeding was among the best remembered. Among 12 to 18 year olds in Montreal, close agreement was noted between mother's reported history and records although actual data were not presented. ${ }^{12}$

\section{BREAST FEEDING/LIPID ASSOCIATIONS}

Blood lipid concentrations are affected by multiple factors, including genetic, environmental, and behavioural. The possibility that infant feeding practices may exert a residual effect on adolescent or adult lipid concentrations is intriguing though complex to sort out analytically. Our findings for plasma cholesterol in 17 year olds parallel those of Marmot et al in 32 year olds ${ }^{1}$ with an inverse association of cholesterol with duration of breast feeding in girls and no consistent finding in boys. The British study, however, compared exclusively breast fed adults with those exclusively bottle fed during the first five months of life whereas we considered the duration of any breast feeding. Prolonged exclusive breast feeding was unusual in our population and at 5 weeks half the babies received complementary milk and at $\mathbf{2}$ to $\mathbf{3}$ months most babies received fruit and vegetables.

Control for confounding variables had little effect on the slope coefficients but tended to reduce the statistical significance of the cholesterol/breast feeding association in girls. The Asian origin, and particularly the North African origin groups, have lower plasma cholesterol concentrations than the Israeli/European origin group, both in this data set and in the entire Jerusalem Lipid Research Clinic Study population ${ }^{4}$ and were breast fed longer. They also have lower fat and saturated fat intake and a higher polyunsaturated to saturated fat ratio. ${ }^{13}$ Whether the dichotomous classification of diverse ethnic groups permits adequate control for the ethnic variable in this small sample can be answered only in larger future studies.

As noted by Marmot $e t$ al there was no consistent association with HDL-cholesterol in our population, and analysis of the association with the proportion of the HDL-cholesterol in total cholesterol showed no additional relationships. ${ }^{1}$ Findings for triglyceride were unexpected and were limited to boys in the regression analysis. This needs to be confirmed.

The small sample in the current study and possible (though unidentified) biases of selection and confounding require caution in interpretation. We view the data as suggesting that further and more extensive study of the association of breast feeding with blood lipids may be fruitful. The consistency with the previous report in adults ${ }^{1}$ lends some support.

Several contradictory reports have been published relating to breast feeding in infancy and subsequent cholesterol concentrations in adulthood in laboratory animals and in childhood in human studies. Reiser $e t$ al based on their animal studies, postulated that an exogenous source of cholesterol early in life may be necessary for development of regulatory mechanisms for normal cholesterol metabolism. ${ }^{14}$ is They suggested that high dietary cholesterol intake soon after birth in mammals establishes a balance in enzyme systems for control of blood cholesterol, that breast milk may contain some additional factor that is involved in the homeostatic control of blood cholesterol and that "the entire normal nursing period is required for normal levels". This hypothesis is supported by several studies in rats ${ }^{16-18}$ but not in others. ${ }^{19}$ In rats dietary cholesterol may affect milk cholesterol concentrations, ${ }^{14}{ }^{16}$ but here too the data are inconsistent. ${ }^{1920}$ Studies of the source of milk cholesterol in goats indicate that, although some milk cholesterol is synthesised in the mammary gland, about $80 \%$ derives from serum cholesterol. ${ }^{21}$

Generally, human data are inconsistent and do not support the Reiser-Sidelman hypothesis. Ziegler and Fomon, however, suggest that published studies may be inadequate in design, analysis, or duration of follow up to put the hypothesis properly to a test. ${ }^{22}$ Serum cholesterol concentrations at 12 months of age were unrelated to earlier cholesterol intake ${ }^{23-25}$; at 12 months, $18-24$ months, and 15-19 years no cholesterol differences were noted in breast fed 
versus low cholesterol formula fed babies ${ }^{26}$; 8 year old boys, but not girls, who were breast fed showed slightly lower cholesterol concentrations ${ }^{22}$; whereas children aged 2.5 years $^{27}$ or 7-11 years ${ }^{28}$ fed with breast or cows' milk had higher serum cholesterol concentrations than those fed a low cholesterol formula; finally, 6 year old girls, though not boys, breast fed for at least three months had significantly lower serum cholesterol concentrations than those fed cows' milk or formula. ${ }^{29}$ The single study that reported data on adolescents ${ }^{26}$ did not seem to take possible sex confounding or sex interactions into account. Both Jerusalem and United States Lipid Research Clinics data show a sex difference in plasma cholesterol concentrations in the 15-19 year age group. ${ }^{40}$

Reiser et al contends that a test of the breast feeding/cholesterol hypothesis requires examination of blood cholesterol in adults rather than in children..$^{15}$ Marmot et al and the current study lend limited support to this notion, and that only among girls. ${ }^{1}$ Cows' milk, the alternative supplement to breast milk in our study population, has similar or somewhat lower cholesterol content than breast milk. ${ }^{28}{ }^{28}$ If the association described is found in future studies it could be surmised that the cholesterol content of milk may not be of central importance but rather some other characteristic associated with breast feeding or breast milk.

The satisfactory validity of the breast feeding history obtained by interview of the mothers over 20 years after birth of their children, and the breast feeding/lipid associations noted in this small sample, suggest that a large interview based investigation of adolescents or young adults should be undertaken.

We thank Dr D Tamir of the Jerusalem Municipality Health Department for access to records from one of the neighbourhood clinics.

\section{References}

${ }^{1}$ Marmot MG, Page CM, Atkins E, Douglas JWB. Effect of breast-feeding on plasma cholesterol and weight in young adults. J Epidemiol Community Health 1980; 34: 164-7.

${ }^{2}$ Kark JD, Slater PE, Stein Y, eds. Cardiovascular risk factors in Jerusalem: the lipid research clinic prevalence study I and II. Isr J Med Sci 1982; 18: 1075-252.

${ }^{3}$ Slater PE, Friedlander Y, Baras M, et al. The Jerusalem lipid research clinc: sampling, response and selected methodological issues. Isr J Med Sci 1982; 18: 1106-12.

'Halfon S-T, Eisenberg S, Baras M, Davies AM, Halperin G, Stein $Y$. Plasma cholesterol, triglyceride and high-density lipoprotein-cholesterol levels in 17-year-old Jerusalem offspring of Jews from 19 countries of birth. Isr J Med Sci 1982; 18: 1121-30.
${ }^{5}$ National Heart, Lung and Blood Institute. Manual of laboratory operations. Lipid and lipoprotein analysis. Bethesda, MD; National Institutes of Health, 1974. (Publication No (NIH) 75-628.)

${ }^{6}$ Fleiss J. Statistical methods for rates and proportions. New York: John Wiley, 1972.

${ }^{7}$ Harlap S, Baras M, Friedlander Y, et al. Contributions of different lipoprotein fractions to variations in total cholesterol between Israeli origin groups and social classes. Isr J Med Sci 1982; 18: 1131-6.

${ }^{8}$ Thaustein J, Halevi HS, Mundel G. Infant feeding practices in Israel. Pediatrics 1960; 26: 321-30.

${ }^{9} \mathrm{Halevi}$ HS. Statistical data on infant feeding and weaning in Israel. Briut Hatzibur 1960; 3: 257-62. (In Hebrew.)

${ }^{10}$ Haggard EA, Brekstad A, Skard AG. On the reliability of the anamnestic interview. Journal of Abnormal and Social Psychology 1960; 61: 311-8.

${ }^{11}$ Robbins LC. The accuracy of parental recall of aspects of child development and of child rearing practices. Journal of Abnormal and Social Psychology 1963; 66: 261-70.

${ }^{12}$ Kramer MS. Do breast-feeding and delayed introduction of solid foods protect against subsequent obesity? $J$ Pediatr 1981; 98: 883-7.

${ }^{13}$ Kaufmann NA, Friedlander Y, Halfon S-T, et al. Nutrient intake in Jerusalem-consumption in 17-year-olds. Isr J Med Sci 1982; 18: 1167-82.

${ }^{14}$ Reiser R, Sidelman Z. Control of serum cholesterol homeostasis by cholesterol in the milk of the suckling rat. J Nutr 1971; 102: 1009-16.

${ }^{15}$ Reiser R, O'Brien BC, Henderson GR, Moore RW. Studies on a possible function for cholesterol in milk. Nutrition Reports International 1979; 19: 835-49.

${ }^{16} \mathrm{Kris}-$ Etherton PM, Layman DK, York PV, Frantz ID. The influence of early nutrition on the serum cholesterol of 0 the adult rat. $J$ Nutr 1979; 109: 276-86.

${ }^{17} \mathrm{Hahn}$ P, Kirby L. Immediate and late effects of premature weaning and of feeding a high fat or carbohydrate diet to weaning rats. $J$ Nutr 1973; 103: 690-6.

${ }^{18}$ Hahn P, Koldovsky O. Late effect of premature weaning on blood cholesterol levels in adult rats. Nutrition Reports International 1976; 13: 87-91.

${ }^{19}$ Hulbron G, Aubert R, Bourgeois F, Lemonnier D. Early cholesterol feeding: are there long-term effects in the rat? J Nutr 1982; 112: 1296-305.

${ }^{20}$ Green $\mathrm{MH}$, Dohner EL, Green JB. Influence of fat and cholesterol on milk lipids and on cholesterol metabolism in the rat. $J$ Nutr 1981; 111: 276-86.

${ }^{21}$ Long CA, Patton S, McCarthy RD. Origins of the cholesterol in milk. Lipids $1980 ; 15$ : 853-7.

${ }^{22}$ Ziegler EE, Fomon SJ. Infant feeding and blood lipid levels during childhood. In: Lauer RM, Shekelle RB, eds. Childhood prevention of atherosclerosis and hypertension. New York: Raven Press, 1980: 121-5.

${ }^{23}$ Glueck CJ, Tsang R, Balistreri W, Fallat R. Plasma and dietary cholesterol in infancy: effects of early low or moderate dietary cholesterol intake on subsequent response to increased dietary cholesterol. Metabolism 1972; 21: 1181-92.

${ }^{24}$ Farris RP, Frank GC, Webber LS, Srinivasan SR, Berenson GS. Influence of milk source on serum lipids and lipoproteins during the first year of life, Bogalusa heart study. Am J Clin Nutr 1982; 35: 42-9.

${ }^{25}$ Darmady JM, Fosbrooke AS, Lloyd JK. Prospective study of serum cholesterol levels during first year of life. $\mathrm{Br}$ Med J 1972; ii: 685-8.

${ }^{28}$ Friedman G, Goldberg SJ. Concurrent and subsequent serum cholesterols of breast- and formula-fed infants. Am J Clin Nutr 1975; 28: 42-5. 
${ }^{27}$ Ward SD, Melin JR, Lloyd FP, Norton JA, Christian JC. Determinants of plasma cholesterol in children-a family study. Am J Clin Nutr 1980; 33: 63-70.

${ }^{28}$ Hodgson PA, Ellefson RD, Elveback LR, Harris LE, Nelson RA, Weidman WH. Comparison of serum cholesterol in children fed high, moderate, or low cholesterol milk diets during neonatal period. Metabolism 1976; 25: 739-46.
${ }^{29}$ Crawford PB, Clark MJ, Pearson DL, Huenemann RL. Serum cholesterol of 6-year-olds in relation to environmental factors. J Am Diet Ass 1981; 78: 41-6. ${ }^{30}$ National Heart, Lung and Blood Institute. The lipid research clinics population studies data book. Vol 1. The prevalence study. Bethesda, MD: National Institutes of Health, 1980. (NIH publication No 80-1527.) 